\title{
Inhibition of osteoclastogenesis and inflammation by phosvitin and phosvitin hydrolysate via NF- $\kappa B$ and MAPK pathways in RAW 264.7 cells
}

\author{
Jiandong Ren ${ }^{\mathrm{a}}$, Subhadeep Chakrabarti ${ }^{\mathrm{b}}$ and Jianping $\mathrm{Wu}^{\mathrm{a}, \mathrm{b}^{*}}$
}

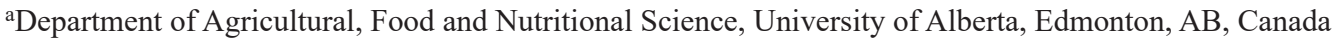

${ }^{\mathrm{b}}$ Cardiovascular Research Centre and Women and Children's Health Research Institute, University of Alberta, Edmonton, AB, Canada

*Corresponding author: Jianping Wu, 4-10 Ag/For Centre, Edmonton, AB, T6G 2P5, Canada. Tel: (780) 492-6885, Fax (780) 492-4265, E-mail:jwu3@ualberta.ca

DOI: $10.31665 /$ JFB.2021.13261

Received: March 27, 2021; Revised received \& accepted: March 31, 2021

Abbreviations: ANOVA, analysis of variance; AP-1, activator protein 1; DMEM, Dulbecco's modified eagle's medium; DTT, dithiothreitol; ELISA, enzyme-linked immunosorbent assay; HPLC, high-performance liquid chromatography; IL, interleukin; iNOS, inducible nitric oxide synthases; JNK, c-Jun N-terminal kinase; LC-MS, liquid chromatography-mass spectrometry; MAPK, mitogen-activated protein kinases; MCP-1, monocyte chemoattractant protein-1; M-CSF, macrophage-colony stimulating factor; NFATc1, nuclear factor of activated T-cells 1; NFкB, nuclear factor kappa beta; OPG, osteoprotegerin; PTH, parathyroid hormone; PV, phosvitin; PVH, phosvitin phosphopeptides; RANK, receptor activator of nuclear factor kappa-B; RANKL, receptor activator of nuclear factor kappa-B ligand; RANTES, regulated on activation, normal T cell expressed and secreted; TNF- $\alpha$, tumor necrosis factor alpha; TRAF6, tumor necrosis factor receptorassociated factor 6; TRAP, tartrate-resistant acid phosphatase

Citation: Ren, J., Chakrabarti, S., and Wu, J. (2021). Inhibition of osteoclastogenesis and inflammation by phosvitin and phosvitin hydrolysate via NF-אB and MAPK pathways in RAW 264.7 cells. J. Food Bioact. 13: 74-81.
\end{abstract}

\begin{abstract}
Phosvitin (PV) is an egg protein. Our recent study showed both phosvitin and phosvitin hydrolysate (PVH) could promote osteoblast differentiation in osteoblast cells. The objective of the study was to investigate the effects of PV and PVH on osteoclastogenesis and possible signalling pathways in RAW264.7 cells. Both PV and PVH inhibited osteoclastogenesis (fewer tartrate-resistant acid phosphatase (TRAP) positive cells and lower TRAP activity), reduced levels of transcription factors, c-Fos and NFATc1 (nuclear factor of activated T-cells, cytoplasmic 1), and suppressed inflammatory biomarkers TNF- $\alpha$ (tumor necrosis factor alpha), MCP-1 (monocyte chemoattractant protein 1), RANTES (regulated on activation, normal T cell expressed and secreted), and inducible nitric oxide synthase. The inhibitory effects of PV and PVH on RAW264.7 cells differentiation were likely mediated through p38, c-Jun N-terminal kinases (JNK) and nuclear factor kappa-light-chain-enhancer of activated B cells (NF-KB) pathways. These results indicated that PV and PVH might inhibit bone resorption activities.
\end{abstract}

Keywords: Phosvitin; Phosvitin hydrolysate; Osteoclast; Bone resorption; Inflammation.

\section{Introduction}

Bone is a metabolic tissue that constantly produces new bones to replace old/damaged bones to maintain a healthy and integrate skeleton system. It is often referred as bone remodeling (Alford et al., 2015). Osteoclast and osteoblast cells play a critical role in bone remodeling by resorbing old bones and secreting new bones, respectively. During bone resporption, mature osteoclasts are formed and start to resorb old bones under stimulation of macrophage-colony stimulating factor (M-CSF) and receptor activator of nuclear factor kappa B-ligand (RANKL). Following resorption, osteoblast progenitors are then recruited on the site of the cavity to form mature osteoblasts and synthesize new bones (Bellido, 2014).

The activities of osteoclasts are essential for bone remodeling 
by resorbing the mineralized bone matrix. Lack of osteoclasts will result in osteopetrosis which can be described as the bone marrow cavity filled with un-resorbed bone tissues (Chen et al., 2018). Osteoclasts are differentiated from monocyte/macrophage lineage and featured with their extraordinary large shape and multiple nucleus. Many factors could affect osteoclast differentiation/activities including calcitriol, parathyroid hormone (PTH) and some inflammatory cytokines (Cappariello et al., 2014). RANKL plays a central role in the regulation of osteoclastogenesis. It can bind the receptor activator of nuclear factor kappa-B (RANK, receptor of RANKL) to stimulate osteoclasts differentiation and maintain their resorption activities by triggering a series of signaling pathways (Honma et al., 2014). Once bound by RANKL, the cytoplasmic domain of RANK recruits tumor necrosis factor (TNF) receptorassociated factor 6 (TRAF6) to trigger a series of cellular reactions involving osteoclast-related proteins including c-Jun N-terminal kinase (JNK), p50/65 nuclear factor kappa-light-chain-enhancer of activated $\mathrm{B}$ cells $(\mathrm{NF}-\kappa \mathrm{B})$ and $\mathrm{p} 38$ mitogen-activated protein kinases (MAPK) (Boyle et al., 2003). Osteoprotegerin (OPG) is a secreted protein that has more affinity to RANKL than RANK thus negatively regulates osteoclastogenesis by binding with RANKL. This RANKL/RANK/OPG interaction dominantly regulates osteoclast differentiation (Martin and Sims, 2015).

It was also well known that many age-related diseases e.g. osteoporosis are associated with inflammation; inflammation results in bone loss when it is associated with diseases such as multiple myeloma, rheumatoid arthritis and periodontal disease (Gibon et al., 2016). In postmenopausal women, estrogen deficiency results in excessive secretion of RANKL in osteoblasts, and RANKL will trigger osteoclasts to produce inflammatory cytokines. Some of these cytokines e.g. interleukin-1 (IL-1), interleukin-6 (IL-6) and tumor necrosis factor-alpha (TNF- $\alpha$ ) could promote osteoclasts differentiation/activities and thus elevate bone resorption (Guo et al., 2016; Sang et al., 2016); other chemokines e.g. monocyte chemoattractant protein 1 (MCP-1) and regulated upon activation normal $\mathrm{T}$ cell expressed and secreted (RANTES), could stimulate osteoclast cell to accelerate its differentiation and resorption activities in an autocrine loop (Kim et al., 2005a; Mulholland et al., 2019).

Food protein and its derived peptides have been widely reported to exert physiological activities (Ambigaipalan and Shahidi, 2017; Ejike et al., 2017). Phosvitin (PV) is a highly phosphorylated protein existing in egg yolk. Phosvitin and phosvitin phosphopeptides have raised considerable research attention due to antioxidant, anti-inflammation, calcium absorption promoting, and bone organogenesis activities (Young et al., 2011; Xu et al., 2012; Hu et al., 2013; Liu et al., 2013; Liu et al., 2017). To investigate the potential of phosvitin, new procedures were developed to extract phosvitin (Ren and Wu 2015) and prepare phosvitin hydrolysate (PVH, rich in phosphopeptides; Ren et al., 2015); using osteoblast cells, we further showed PV and PVH promoted osteoblast differentiation and arrested production of anti-inflammatory cytokines by regulating ERK and AKT signaling (Ren et al., 2019). Given the critical roles of osteoclasts played in bone remodelling and inflammation, it is necessary to investigate the role of PV/PVH on osteoclastogenesis and possible RANK-mediated signalling pathways in osteoclasts.

\section{Materials and Methods}

\subsection{Chemicals and reagents}

Dulbecco's Modified Eagle's medium (DMEM) (11995-065), penicillin-streptomycin (15140122), fetal bovine serum (FBS; A31605) and Alamar blue (DAL1025) were manufactured by Thermo Fisher Scientific (Thermo Fisher Scientific Inc., Carlsbad, CA, USA). Triton-X-100 (97062-208) was manufactured by VWR International (VWR International Inc., West Chester, PA, USA). RANKL (462-TEC-010) was manufactured by R\&D Systems Inc. (R\&D Systems Inc., Minneapolis, MN, USA). Primary antibodies JNK (mouse monoclonal, cat\# mab2076), p-JNK (rabbit monoclonal, cat\# mab1205) were purchased from R\&D Systems Inc. (Minneapolis, MN, USA); phospho-p65 (rabbit polyclonal, cat\# sc-3033), p65 (mouse monoclonal, cat\# sc-8008) were purchased from Santa Cruz Biotechnology Inc. (Santa Cruz, CA, USA); NFATc1 (rabbit monoclonal, cat\# mab8032) was purchased from Cell Signaling Technology Inc. Danvers, MA, USA); inducible nitric oxide synthase (iNOS) (rabbit polyclonal, cat\# ab-3523), and $\alpha$-tubulin (rabbit polyclonal, cat\# ab15246) were purchased from Abcam Inc. (Cambridge, MA, USA); Phospho-p38 (p-p38) (rabbit polyclonal, cat\# NB500-138), c-Fos (rabbit polyclonal, cat\# NB110) were purchased from Novus Biologicals Inc. (Littleton, CO, USA); Goat anti-rabbit (925-68071) and Donkey anti-mouse (926-32212) fluorochrome-conjugated secondary antibodies were purchased from Licor Biosciences (Lincoln, NB, USA).

Phosvitin was prepared according to Ren and Wu (2015) with $88.0 \%$ purity determined by chromatography; PVH was prepared by pancreatin (p7545-25G; Sigma-Aldrich, Ltd., Oakville, ON, Canada) hydrolysis and the characterization information about this product has been reported by our previous research (Ren et al., 2015).

\subsection{Cell culture}

The RAW 264.7 cells (TIB-71; American Type Culture Collection, Manassas, VA, USA) were cultured as previously described (Ren et al., 2019). Briefly cells were grown in DMEM. RANKL (50 $\mathrm{ng} / \mathrm{mL}$ ) was added to induce osteoclastogenesis while PV or PVH were added for different analysis as described in the following sections. Alamar blue is a fluorescence dye and used to determine the toxicity as per manufacturer's manual. Briefly, RAW 264.7 cells were incubated with PV/PVH at $50-500 \mu \mathrm{g} / \mathrm{ml}$ for $72 \mathrm{hr}$ before $20 \mu \mathrm{L}$ Alamar blue was added for incubation of another $4 \mathrm{hr}$. The readings with excitation of $560 \mathrm{~nm}$ and emission of $590 \mathrm{~nm}$ were recorded. No significant difference was detected between $\mathrm{PV} / \mathrm{PVH}$ treated cells and untreated cells at the concentrations used in experiments (Figure s1).

\subsection{Determination of tartrate-resistant acid phosphatase (TRAP) activity and staining of TRAP positive cells}

RAW264.7 cells were cultured as described by Rahman et al. (2006). Briefly RAW264.7 cells were treated with $50 \mathrm{ng} / \mathrm{ml}$ RANKL for $24 \mathrm{~h}$ to induce osteoclastogenesis, and then 50, 100 and $500 \mu \mathrm{g} / \mathrm{ml} \mathrm{PV/PVH}$ were added. At the end of day 5, cell lysate was collected to test TRAP activity by using Sigma's TRAP kit (CS0740) following the manufacturer's instruction. Absorbance at $405 \mathrm{~nm}$ was measured by using a Molecular Devices SpectraMax M3 microplate reader (Molecular Devices LLC, San Jose, CA, USA). In another experiment, cells were not lysed but stained with Sigma's TRAP staining kit (387A-1KT) following the manufacturer's instruction. Morphology of the cells were captured by an Axiocam 105 color camera and processed by using Zen 2 software on a Zeiss Primovert microscope system (Carl Zeiss Microscopy LLC, White Plains, NY, USA). 


\subsection{Preparation of cell lysate for RANKL induced signaling path- ways study}

RAW264.7 cells were cultured as previously described (Rahman et al., 2006). To study the effects of PV/PVH on signaling pathway, cells were treated with PV or PVH $(500 \mu \mathrm{g} / \mathrm{ml})$ for $48 \mathrm{hr}$ and then RANKL $(50 \mathrm{ng} / \mathrm{ml})$ for another $30 \mathrm{~min} \mathrm{NF- \kappa B}$ pathway, or $45 \mathrm{~min}$ for mitogen-activated protein kinase (MAPK) pathway. To study the changes in nuclear factors of activated T-cells, cytoplasmic 1 (NFATc1) and cFos, cells were incubated with RANKL $(50 \mathrm{ng} / \mathrm{ml})$ for $24 \mathrm{hr}$ and then $\mathrm{PV} / \mathrm{PVH}(500 \mu \mathrm{g} / \mathrm{ml})$ for another 3 days. Cell lysate was collected as previously described (Chakrabarti and Davidge, 2016).

\subsection{Inflammatory cytokines/chemokines in RANKL stimulated RAW264.7 cells by enzyme-linked immunosorbent assay (ELISA)}

RAW264.7 cells $\left(1 \times 10^{5}\right.$ cells/well) were cultured in a 24 -well plate for $24 \mathrm{hr}$, followed by treatment with $\mathrm{PV} / \mathrm{PVH}$ for another $24 \mathrm{hr}$, and then RANKL $(50 \mathrm{ng} / \mathrm{ml})$ to stimulate the production of cytokines/chemokines. At day 3, the medium was collected for analysis of tumor necrosis factor alpha (TNF- $\alpha$ ), regulated on activation, normal $\mathrm{T}$ expressed and secreted (RANTES) chemokine and monocyte chemoattractant protein-1 (MCP-1) by their respective kits. RANTES ELISA kit (MMR00), TNF- $\alpha$ ELISA kit (NBP1-92681) and MCP-1 ELISA kit (KA1831) were purchased from R\&D Systems, Inc. (Minneapolis, MN, USA).

\subsection{Western blot}

The cell lysate from section 2.4 was subjected to Western blot according to a previously published method (Chakrabarti and Davidge, 2016). Briefly, proteins were first separated by sodium dodecyl sulphate-polyacrylamide gel electrophoresis (SDS-PAGE) and then incubated with various primary antibodies against $\mathrm{JNK}$, p-JNK, phospho-p65, p65, NFATc1, iNOS, Phospho-p38 (p-p38), cFos, and $\alpha$-tubulin. After further incubation with fluorochrome-conjugated secondary antibodies, the images of cellular proteins were captured and densitometry was quantified by using a Licor Odyssey BioImager (Licor Biosciences, Lincoln, NB, USA) equipped with the Image Studio Lite 5.2 software (Licor Biosciences, Lincoln, NB, USA ). The densitometry of treated groups was compared to that of untreated control and presented as a percentage of the latter.

\subsection{Statistics}

Three independent experiments were carried out and the results were expressed as mean \pm standard error of the mean. Data was processed by PRISM 6 statistical software (GraphPad Software, San Diego, CA) using one way analysis of variance (ANOVA) with Dunnett's post-hoc test to determine the effects of treatments and to generate graphs. A $p<0.05$ indicates significant effect was detected.

\section{Results and Discussion}

\subsection{Effects of PV/PVH on RANKL-stimulated RAW264.7 cells differentiation}

RANKL is widely used to differentiate RAW264.7 cells into tar- trate-resistant acid phosphatase (TRAP)-positive osteoclast-like cells. TRAP activity is commonly used to evaluate osteoclasts' functions (Shevde et al., 2000). Effects of PV/VPP on the TRAP activities were shown in Figure 1a and b. A group of representative images under each treatment was shown in Figure 1c.

The TRAP activity of RAW264.7 cells was not affected by $\mathrm{PV} / \mathrm{PVH}$ at concentrations of 50 and $100 \mu \mathrm{g} / \mathrm{ml}$, but was significantly suppressed at a concentration of $500 \mu \mathrm{g} / \mathrm{ml}$ (Figure 1a, b). RAW264.7 cells differentiated into large, multi-nucleated osteoclast cells (stained with purple color) in the presence of RANKL alone; the differentiation was not affected by adding PV/PVH at low concentrations $(50 \mu \mathrm{g} / \mathrm{ml})$, but was significantly suppressed at higher concentrations of 100 and $500 \mu \mathrm{g} / \mathrm{ml}$ (Figure 1c). The presence of mononuclear RAW264.7 cells stained as TRAP positive indicated that these cells were activated but not differentiated.

RANKL-RANK interaction plays a central role in regulating osteoclasts' signalling pathways and expression of transcription factors including nuclear factor of activated T-cells, cytoplasmic 1 (NFATc1) and c-Fos, two critical transcription factors for osteoclastogenesis. NFATc1 is the master transcription regulator for osteoclastogenesis, regulating osteoclast specific genes such as TRAP, cathepsin $\mathrm{K}$ and calcitonin receptor etc. (Takayanagi et al., 2002; Kim et al., 2005b). The mRNA expression of NFATc1 is selectively regulated by RANKL (Asagiri et al., 2005). Monocytes and macrophages are the precursors of osteoclasts. The role of c-Fos is to commit these precursors into osteoclasts instead of mature macrophages through the formation of activation protein 1 (AP1) (Teitelbaum, 2000). Besides, c-Fos is essential for the autoamplification of NFATc 1 as NFATc 1 expression was completely inhibited in cells lack of c-Fos (Matsuo et al., 2004). In this experiment, the presence of RANKL stimulated osteoclastogenesis and therefore upregulated the expression of NFATc1 and c-Fos; however, the treatment with PV/ PVH significantly downregulated these two transcription factors to the level of untreated cells (Figure 2). These results agree well with the observation in TRAP activity assay, and further confirmed the inhibitory effects of PV/PVH on osteoclast differentiation.

\subsection{Effects of PV/PVH on inflammatory response in RANKL stim- ulated RAW264.7 cells}

RAW264.7 is a macrophage-like cell line and a frequently used model for inflammation studies. In this experiment, addition of RANKL at $50 \mathrm{ng} / \mathrm{ml}$ not only induced osteoclastogenesis but also elevated inflammatory response. The production of inducible nitric oxide synthase (iNOS), tumor necrosis factor alpha (TNF- $\alpha$ ), regulated on activation, normal $\mathrm{T}$ expressed and secreted (RANTES) and monocyte chemoattractant protein-1 (MCP-1) were all elevated by RANKL treatment. Adding $500 \mu \mathrm{g} / \mathrm{ml}$ PV/PVH however significantly reduced the production of these inflammation products, which supported the anti-inflammation activity of PV/PVH during osteoclast differentiation (Figure 3). One major function of iNOS is to catalyze the production of NO (Surh et al., 2001), which is one of the key factors to maintain the physiological activities of osteoclasts. Thus downregulation of iNOS might result in reduced osteoclast activities (Brandi et al., 1995). Many inflammatory cytokines could cause bone loss in rheumatoid arthritis (Tracey et al., 2008). It was reported that excessive TNF- $\alpha$ formation might have negative effects on bone remodelling by stimulating osteoblast to express RANKL and directly working on osteoclasts to accelerate bone resorption (Kim et al., 2005a). RANTES and MCP-1 were 

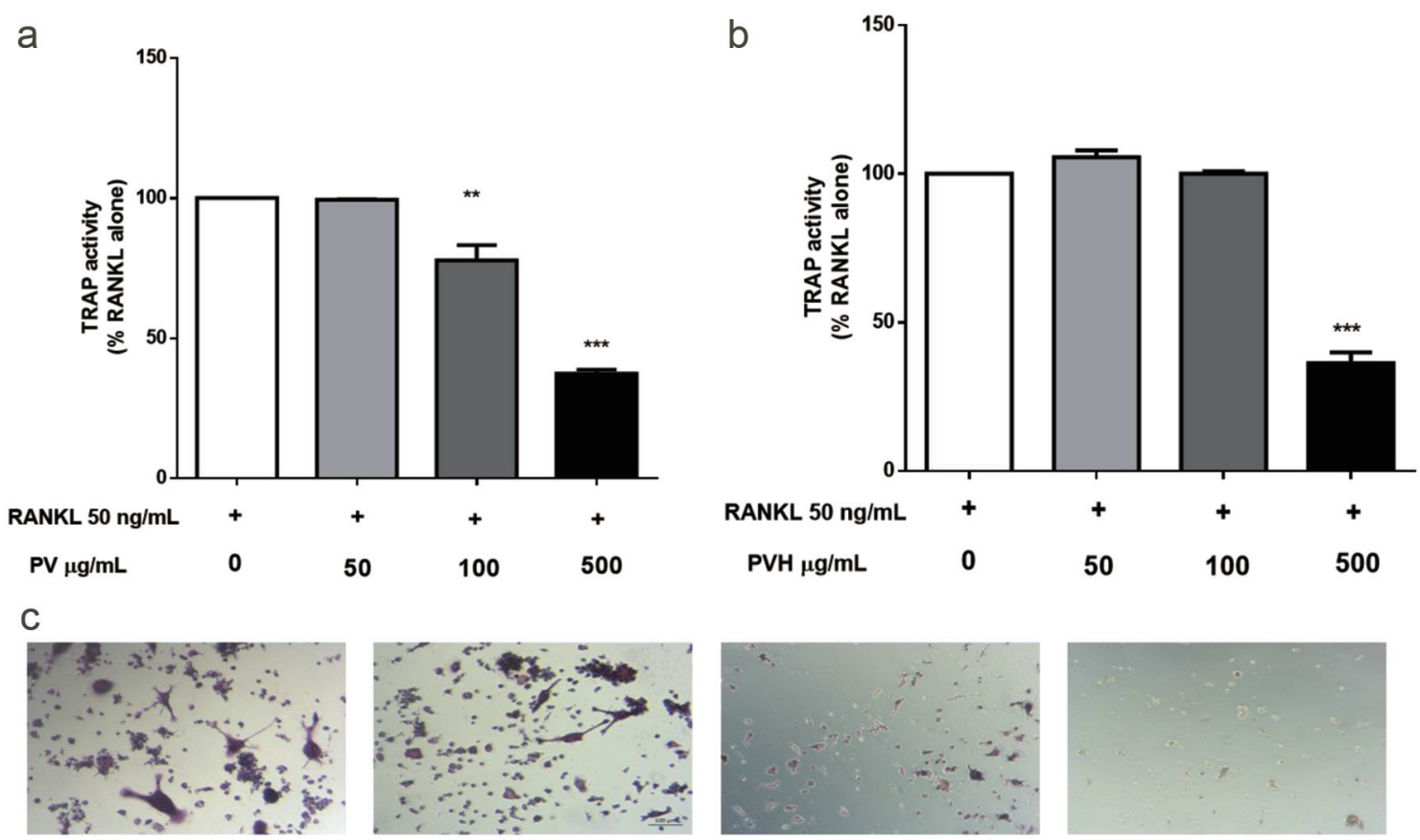

RL alone
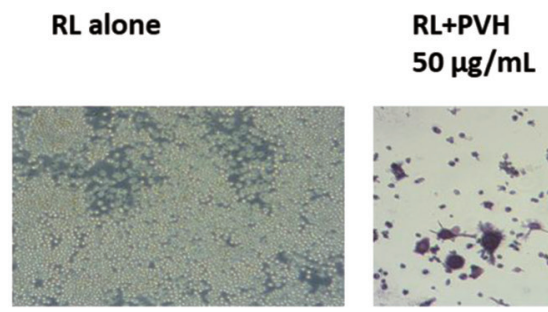

No RL

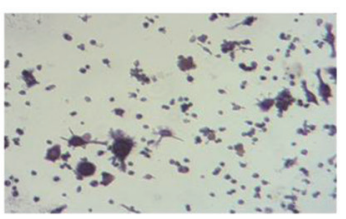

RL+PV

$50 \mu \mathrm{g} / \mathrm{mL}$
RL+PVH $100 \mu \mathrm{g} / \mathrm{mL}$

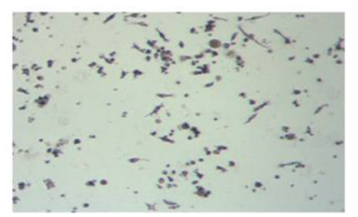

RL+PV $100 \mu \mathrm{g} / \mathrm{mL}$

\section{RL+PVH $500 \mu \mathrm{g} / \mathrm{mL}$}

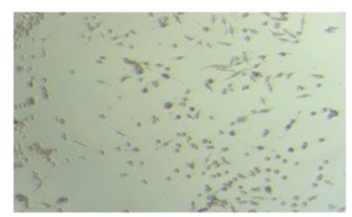

RL+PV $500 \mu \mathrm{g} / \mathrm{mL}$

Figure 1. Effects of PV/PVH on the formation of TRAP-positive cells in RANKL-stimulated RAW264.7 cells. RAW264.7 cells were incubated with 50 ng/ml RANKL for $24 \mathrm{hr}$ followed by PV or PVH for another 5 days, then the cells were lysed by $0.2 \%$ Triton X-100. TRAP activity in cell lysate was determined by using Sigma's TRAP kit (CS0740). (a) TRAP activity of PV treated RAW264.7 cells; (b) TRAP activity of PVH treated RAW264.7 cells, (c) A set of representative images of cells stained with Sigma's TRAP staining kit $(387 \mathrm{~A}-1 \mathrm{KT})$. Results were expressed as the mean \pm standard error of the mean $(\mathrm{n}=3)$. $*, * *$ and $* * *$ indicate $p<0.05, p<0.01$ and $p<0.001$ respectively, as compared to the group of RANKL alone. (RL, RANKL; PV, phosvitin; PVH, phosvitin hydrolysate)

produced when osteoclast precursor differentiated into mature osteoclasts; the released RANTES and MCP-1 also stimulated osteoclast differentiation and formed an autocrine loop (Kim et al., 2005a; Mulholland et al., 2019). PV/PVH have been reported to exert anti-inflammation activities with other cell lines (Young et al., 2011; Xu et al., 2012; Hu et al., 2013). The results from our experiment suggested $\mathrm{PV} / \mathrm{PVH}$ also possessed anti-inflammation ability in RANKL stimulated osteoclast differentiation which might have potential implications in the studies on inflammation related bone diseases.

\subsection{Effects of PV/PVH on signalling pathways in RAW264.7 cells}

Next, we studied the effects of PV/PVH on the signalling pathways involved with RANKL-induced osteoclastogenesis. The RANKLRANK interaction triggered cytoplasmic domain of RANK to recruit tumor necrosis factor (TNF) receptor-associated factor 6 (TRAF6), and then two important osteoclast-related signaling pathways, p50/65 nuclear factor kappa-light-chain-enhancer of activated $\mathrm{B}$ cells $(\mathrm{NF}-\kappa \mathrm{B})$ and mitogen-activated protein kinases (MAPKs), are activated afterwards, associated with regulating osteoclastogenesis (Boyle et al., 2003).

The MAPK p38 and c-Jun N-terminal protein kinase (JNK) pathways are critical for osteoclastogenesis as the either JNK or p38 MAPK inhibitor could inhibit the differentiation (Takayanagi et al., 2002; Ikeda et al., 2004). Studies suggested that p38 pathway was essential for RANKL-induced osteoclast differentiation, but not for RANKL-induced osteoclast function (Li et al., 2002). JNK is a type of serine/threonine kinases with a wide spectrum of functions to support cell growth, differentiation, and apoptosis (Zhang and Liu, 2002). In osteoclasts, RANKL elevates the expression of c-Jun, which binds c-Fos to form activator protein 1 (AP-1) and thus regulates NFATc1 expression via AP-1 level (Asagiri et al., 2005).

In this experiment, RANKL treatment elevated the levels of phosphor-p38 and phosphor-JNK in RAW264.7 cells. Pre-treatments with PV/PVH for 48 hours significantly reduced the levels 
a

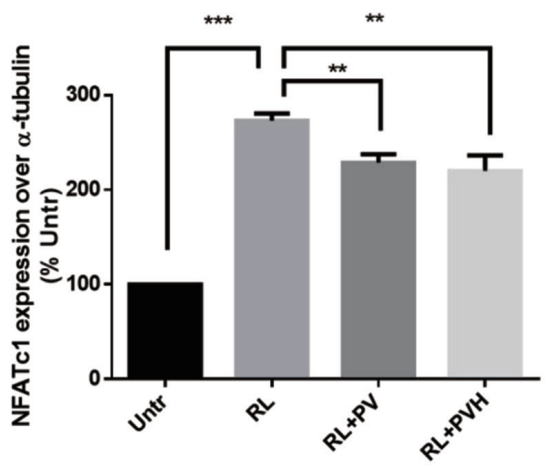

NFATC1

$\alpha$-Tubulin b

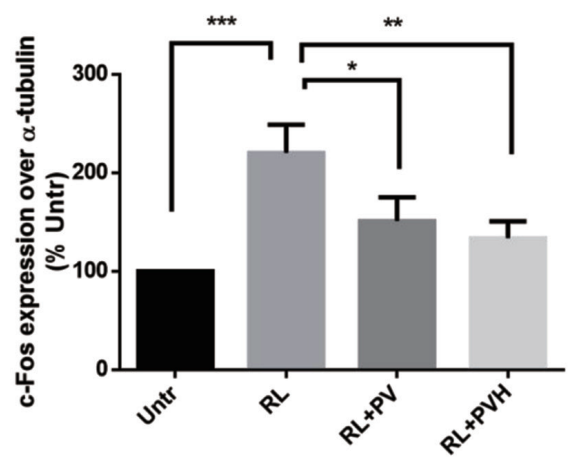

c-Fos

$\alpha$-Tubulin

Figure 2. Effects of PV/PVH on osteoclastogenesis transcription factors in RANKL-stimulated RAW264.7 cells. RAW264.7 cells were cultured with RANKL $(50 \mathrm{ng} / \mathrm{ml})$ for $24 \mathrm{hr}$ and followed by PV $(500 \mu \mathrm{g})$ or PVH $(500 \mu \mathrm{g})$ for another $72 \mathrm{hr}$. A: Whole cell lysates were analyzed by Western blot using antibodies against NFATc1. A set of representative images was shown. B: Whole cell lysates were analyzed by Western blot using antibodies to c-Fos. A set of representative images was shown. Results were expressed as the mean \pm standard error of the mean $(\mathrm{n}=3)$. *, ** and $* * *$ indicate $p<0.05, p<0.01$ and $p<$ 0.001 respectively, as compared to the group of RANKL alone. (Untr, untreated group without RANKL or PV/PVH; RL, RANKL; PV, phosvitin; PVH, phosvitin hydrolysate)
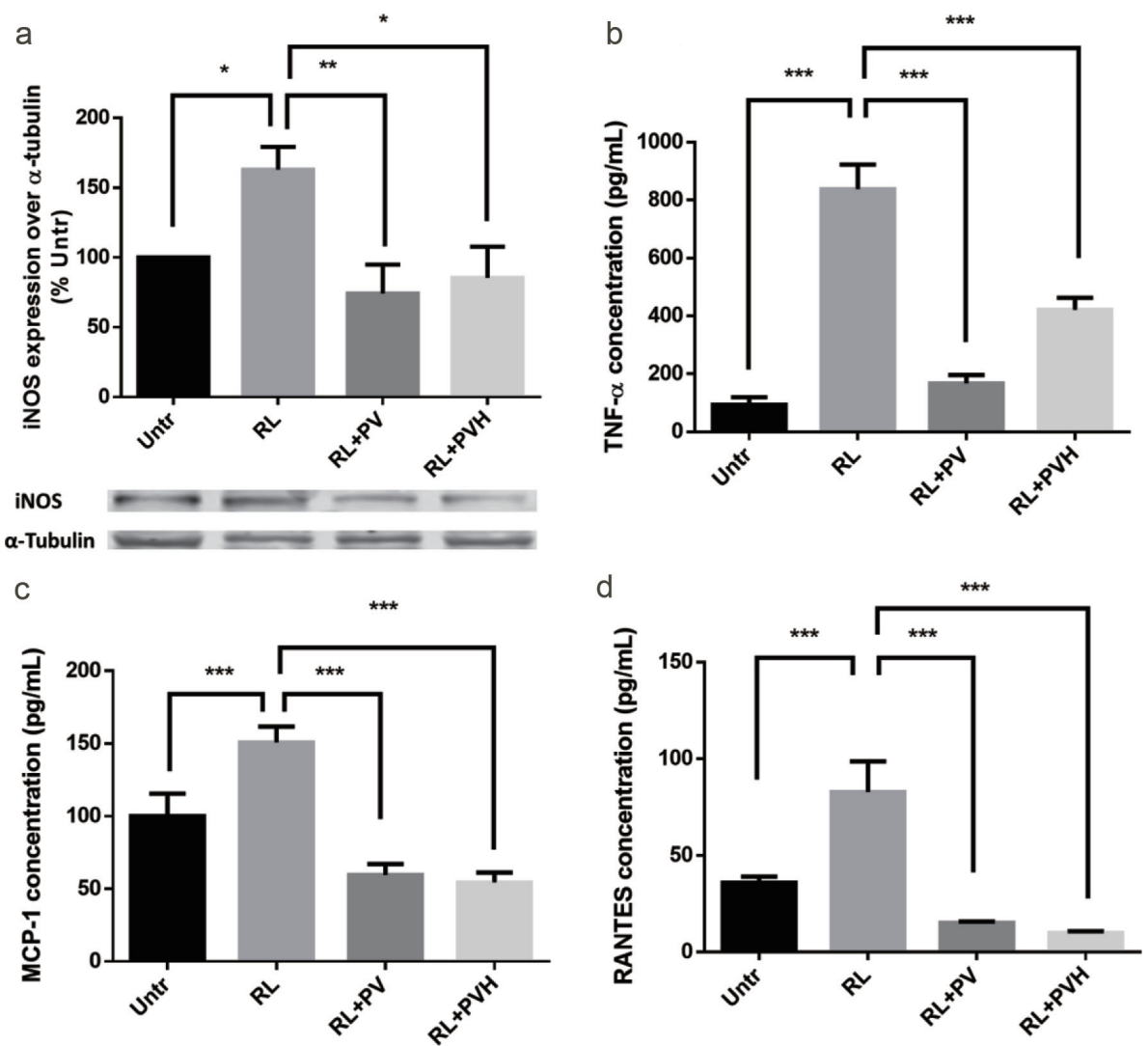

Figure 3. Effects of PV/PVH on inflammation protein expression/secretion in RANKL-stimulated RAW264.7 cells. RAW264.7 cells were cultured for 24 hr. Then, cells were incubated with either PV $(500 \mu \mathrm{g})$ or PVH $(500 \mu \mathrm{g})$ for $24 \mathrm{hr}$. RANKL $(50 \mathrm{ng} / \mathrm{ml})$ was added to stimulate inflammatory cytokines/chemokines secretion for $16 \mathrm{hr}$. At the end of culture, medium was collected and analyzed by using corresponding ELISA kits or the cell lysates were analyzed by Western blot. (a) Whole cell lysates were analyzed by Western blot using antibodies against iNOS. A set of representative images was shown. (b) TNF- $\alpha$ secretion in cell medium. (c) MCP-1 secretion in cell medium. (d) RANTES secretion in cell medium. Results were expressed as the mean \pm standard error of the mean ( $\mathrm{n}$ $=3$ ). ${ }^{*}, * *$ and $* * *$ indicate $p<0.05, p<0.01$ and $p<0.001$ respectively, as compared to the group of RANKL alone. (Untr, untreated group without RANKL or PV/PVH; RL, RANKL; PV, phosvitin; PVH, phosvitin hydrolysate) 

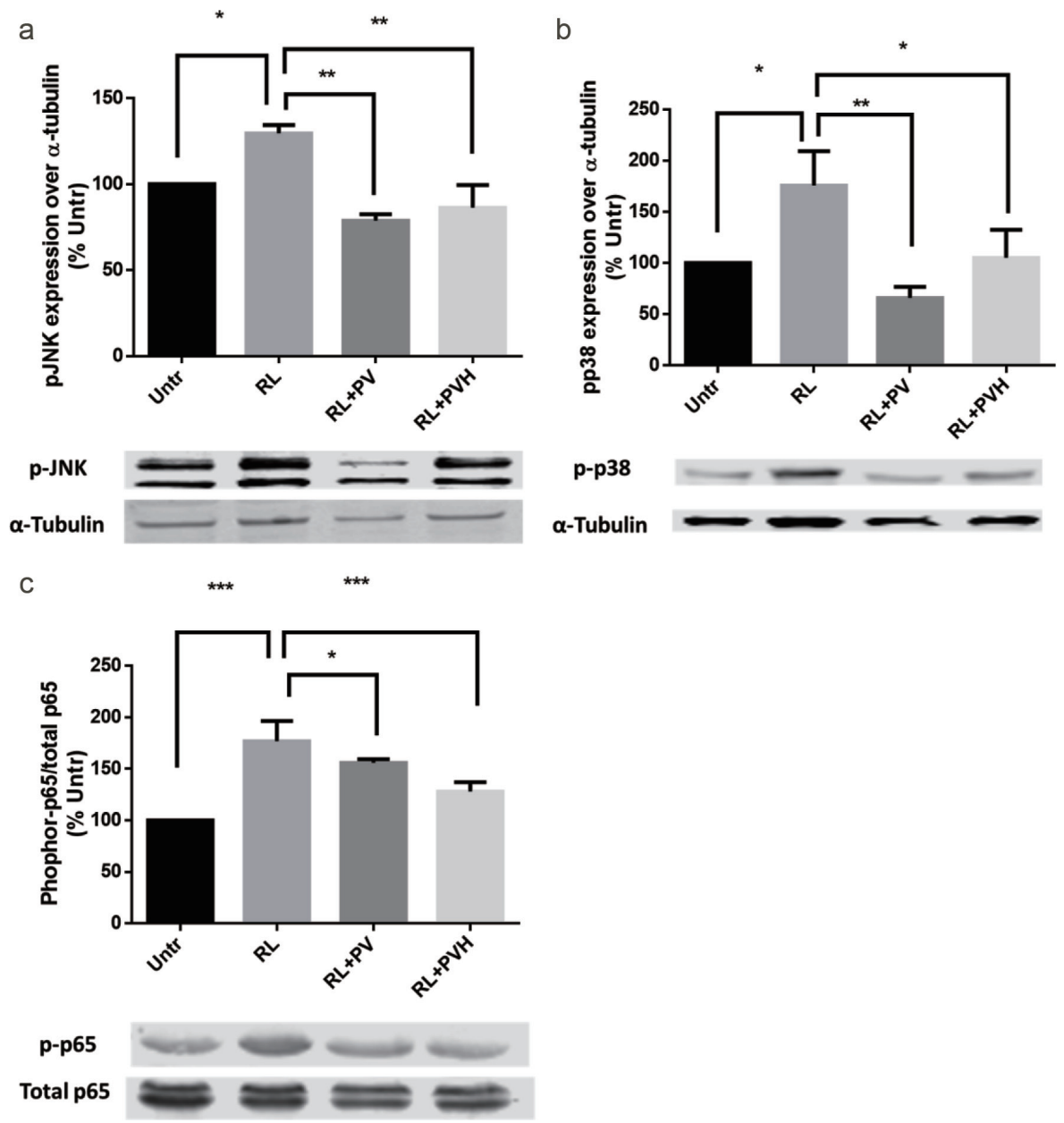

Figure 4. Effects of PV/PVH on different signaling pathways in RANKL-stimulated RAW264.7 cells. RAW264.7 cells were incubated with either PV (500 $\mu \mathrm{g}$ ) or PVH $(500 \mu \mathrm{g})$ for $48 \mathrm{hr}$, followed by treatment with RANKL $(50 \mathrm{ng} / \mathrm{ml})$ for $30 \mathrm{~min}$ for JNK/P36, or $45 \mathrm{~min}$ for P-65 expression. (a) Whole cell lysates were analyzed by Western blot using antibodies against JNK and phosphor-JNK. A set of representative images was shown. (c) Whole cell lysates were analyzed by Western blot using antibodies against p38 and phosphor-p38. A set of representative images was shown. (a) Whole cell lysates were analyzed by Western blot using antibodies against P65 and phosphor-P65. A set of representative images was shown. Results were expressed as the mean \pm standard error of the mean $(\mathrm{n}=3)$. $* * *$ and $* * *$ indicate $p<0.05, p<0.01$ and $p<0.001$ respectively as compared to the group of RANKL alone. (Untr, untreated group without RANKL or PV/PVH; RL, RANKL; PV, phosvitin; PVH, phosvitin hydrolysate)

of phosphor-p38 and phosphor-JNK (Figure 4a, b). These results indicated PV/PVH could inhibit RANKL-induced p38 and JNK activation.

$\mathrm{NF}-\kappa \mathrm{B}$ includes a series of transcription factors that target at a specific gene fragment known as $\kappa \mathrm{B}$. These transcription factors are named as Rel (cRel), RelA(p65), RelB, NF-kappaB1 (p50) and NF-kappaB2 (p52) respectively. In the classical pathway of NF$\kappa B$, RANKL induces the formation of active subunit of p65/p50 and translocation to nucleus to change gene transcription (Hayden and Ghosh, 2004). The transcription factor of NFATc1 is also regulated by NF- $\kappa$ B pathway since it was observed that NF- $\kappa$ B inhibitor could reduce NFATc1 expression in RANKL stimulated osteoclasts (Takatsuna et al., 2005). NF- $\kappa B$ is also a classical pathway that regulates cellular response to a stimulus (Gilmore, 2006). NF$\kappa \mathrm{B}$ pathway mediated iNOS expression has been widely reported (Surh et al., 2001). RANKL induced inflammatory responses in our experiments could be explained as RANKL activated NF$\kappa \mathrm{B}$ pathway. RANKL elevated NF- $\kappa \mathrm{B}$ phosphor-p65 expression, while adding PV/PVH significantly reduced phosphor-p65 expres- sion especially for PVH (Figure 4c). This observation indicated that $\mathrm{PV} / \mathrm{PVH}$ treatment suppressed the NF- $\mathrm{\kappa B}$ pathway, leading to the reduced level of NFATc1 as well as the inflammatory markers shown in Figure 3.

\section{Conclusions}

We reported that both PV and PVH reduced TRAP activity and expression of transcription factors of NFATc1/c-Fos in RANKLinduced RAW264.7 cells, indicating an inhibitory role of PV/ PVH RANKL-induced osteoclastogenesis. PV/PVH treatments also supressed the secretion of inflammatory cytokines/chemokine (TNF- $\alpha$, MCP-1, RANTES) and expression of iNOS. The inhibitory effects of PV/PVH on RANKL-induced osteoclastogenesis and inflammatory response were probably mediated through MAPK JNK/p38 or NF-אB p65 pathways, or both. Our results suggested the potential of PV and PVH for use in the prevention of osteoporosis. 


\section{Supplementary Material}

Figure S1. Cell viability of PV/PVH treated RAW 264.7 cells.Cells were incubated in a 96 well tissue culture plate with $\mathrm{PV} / \mathrm{PVH}$ at $50,100,500 \mu \mathrm{g} / \mathrm{ml}$ for 5 days (with medium change on day 3 ) and then $20 \mu \mathrm{L}$ Alamar blue for another $4 \mathrm{hr}$. The fluorescence of the medium was read with excitation wavelength of $560 \mathrm{~nm}$ and emission wavelength of $590 \mathrm{~nm}$. Results were expressed as the mean \pm standard error of the mean $(n=4)$.

\section{Acknowledgments}

This research was supported by grants from Alberta Agriculture and Forestry, Egg Farmers of Canada, Michael Foods Ltd., and Natural Sciences and Engineering Research Council (NSERC) of Canada to J. Wu. The funders had no role in the study design, data collection and analysis, decision to publish or preparation of the manuscript.

\section{Conflict of interest}

None.

\section{Author contributions}

Jiandong Ren designed the study, collected and analyzed data, interpreted the results, and drafted the manuscript. Jianping Wu contributed to the study design and critical revision of the manuscript. Subhadeep Chakrabarti contributed to the study design, and revision of the manuscript. All authors approved the final version of the manuscript. None.

\section{References}

Alford, A.I., Kozloff, K.M., and Hankenson, K.D. (2015). Extracellular matrix networks in bone remodeling. Int. J. Biochem. Cell Biol. 65: 20-31.

Ambigaipalan, P., and Shahidi, F. (2017). Bioactive peptides from shrimp shell processing discards: Antioxidant and biological activities. J. Funct. Foods 34: 7-17.

Asagiri, M., Sato, K., Usami, T., Ochi, S., Nishina, H., Yoshida, H., and \& Takayanagi, H. (2005). Autoamplification of NFATc1 expression determines its essential role in bone homeostasis. J. Exp. Med. 202(9): 1261-1269.

Bellido, T. (2014). Osteocyte-driven bone remodeling. Calcif. Tissue Int. 94(1): 25-34.

Boyle, W.J., Simonet, W.S., and Lacey, D.L. (2003). Osteoclast differentiation and activation. Nature 423(6937): 337.

Brandi, M.L., Hukkanen, M., Umeda, T., Moradi-Bidhendi, N., Bianchi, S., Gross, S.S., and \& MacIntyre, I. (1995). Bidirectional regulation of osteoclast function by nitric oxide synthase isoforms. Proc. Natl. Acad. Sci. 92(7): 2954-2958.

Cappariello, A., Maurizi, A., Veeriah, V., and Teti, A. (2014). The great beauty of the osteoclast. Arch. Biochem. Biophys. 558: 70-78.

Chakrabarti, S., and Davidge, S.T. (2016). Analysis of G-protein coupled receptor 30 (GPR30) on endothelial inflammation. In: Eyster, K.M. (Ed.). Estrogen Receptors. Humana Press, New York, pp. 503-516.

Chen, X., Wang, Z., Duan, N., Zhu, G., Schwarz, E.M., and Xie, C. (2018). Osteoblast-osteoclast interactions. Connect. Tissue Res. 59(2): 99-107.

Ejike, C.E., Collins, S.A., Balasuriya, N., Swanson, A.K., Mason, B., and Udenigwe, C.C. (2017). Prospects of microalgae proteins in producing peptide-based functional foods for promoting cardiovascular health. Trends Food Sci. Technol. 59: 30-36.

Gibon, E., Lu, L., and Goodman, S.B. (2016). Aging, inflammation, stem cells, and bone healing. Stem Cell Res. Ther. 7(1): 44.

Gilmore, T.D. (2006). Introduction to NF-KB: players, pathways, perspectives. Oncogene 25(51): 6680.

Guo, S., Ni, Y., Ben, J., Xia, Y., Zhou, T., Wang, D., and Chen, Q. (2016). Class a scavenger receptor exacerbates osteoclastogenesis by an Interleukin-6-mediated mechanism through ERK and JNK signaling pathways. Int. J. Biol. Sci. 12(10): 1155.

Hayden, M.S., and Ghosh, S. (2004). NF-kB and Rel proteins: evolutionarily conserved mediators of immune responses. Genes Dev. 18: 21952224.

Honma, M., Ikebuchi, Y., Kariya, Y., and Suzuki, H. (2014). Regulatory mechanisms of RANKL presentation to osteoclast precursors. Curr. Osteoporos. Rep. 12(1): 115-120.

Hu, L., Sun, C., Wang, S., Su, F., and Zhang, S. (2013). Lipopolysaccharide neutralization by a novel peptide derived from phosvitin. Int. J. Biochem. Cell Biol. 45(11): 2622-2631.

Ikeda, F., Nishimura, R., Matsubara, T., Tanaka, S., Inoue, J.I., Reddy, S.V., and Kukita, T. (2004). Critical roles of c-Jun signaling in regulation of NFAT family and RANKL-regulated osteoclast differentiation. J. Clin. Invest. 114(4): 475-484.

Kim, N., Kadono, Y., Takami, M., Lee, J., Lee, S.H., Okada, F., and Yeh, W.C. (2005). Osteoclast differentiation independent of the TRANCERANK-TRAF6 axis. J. Exp. Med. 202(5): 589-595.

Kim, Y., Sato, K., Asagiri, M., Morita, I., Soma, K., and Takayanagi, H. (2005). Contribution of nuclear factor of activated T cells $\mathrm{c} 1$ to the transcriptional control of immunoreceptor osteoclast-associated receptor but not triggering receptor expressed by myeloid cells-2 during osteoclastogenesis. J. Biol. Chem. 280(38): 32905-32913.

Li, X., Udagawa, N., Itoh, K., Suda, K., Murase, Y., Nishihara, T., and Takahashi, N. (2002). p38 MAPK-mediated signals are required for inducing osteoclast differentiation but not for osteoclast function. Endocrinology 143(8): 3105-3113.

Liu, J., Czernick, D., Lin, S.C., Alasmari, A., Serge, D., and Salih, E. (2013). Novel bioactivity of phosvitin in connective tissue and bone organogenesis revealed by live calvarial bone organ culture models. Dev. Biol. 381(1): 256-275.

Liu, Q., Li, C., Geng, F., Huang, X., and Ma, M. (2017). Hen egg yolk phosvitin stimulates osteoblast differentiation in the absence of ascorbic acid. J. Sci. Food Agric. 97(13): 4532-4538.

Martin, T.J., and Sims, N.A. (2015). RANKL/OPG; critical role in bone physiology. Rev. Endocr. Metab. Disord. 16(2): 131-139.

Matsuo, K., Galson, D.L., Zhao, C., Peng, L., Laplace, C., Wang, K.Z., and Wagner, E.F. (2004). Nuclear factor of activated T-cells (NFAT) rescues osteoclastogenesis in precursors lacking c-Fos. J. Biol. Chem. 279(25): 26475-26480.

Mulholland, B.S., Forwood, M.R., and Morrison, N.A. (2019). Monocyte chemoattractant protein-1 (MCP-1/CCL2) drives activation of bone remodelling and skeletal metastasis. Curr. Osteoporos. Rep. 17(6): 538-547.

Rahman, M.M., Bhattacharya, A., and Fernandes, G. (2006). Conjugated linoleic acid inhibits osteoclast differentiation of RAW264. 7 cells by modulating RANKL signaling. J. Lipid Res. 47(8): 1739-1748.

Ren, J., Chakrabarti, S., and Wu, J. (2019). Phosvitin and its hydrolysate promote differentiation and inhibit TNF-a induced inflammation in MC3T3-E1 cells via ERK and AKT pathways. J. Funct. Foods 53: 259265.

Ren, J., Li, Q., Offengenden, M., and Wu, J. (2015). Preparation and characterization of phosphopeptides from egg yolk phosvitin. J. Funct. Foods 18: 190-197.

Ren, J., and Wu, J. (2015). Thermal-aided phosvitin extraction from egg yolk. J. Sci. Food Agric. 95(13): 2595-2600.

Sang, C., Zhang, Y., Chen, F., Huang, P., Qi, J., Wang, P., and Guo, L. (2016) Tumor necrosis factor alpha suppresses osteogenic differentiation of MSCs by inhibiting semaphorin 3B via Wnt/ $\beta$-catenin signaling in estrogen-deficiency induced osteoporosis. Bone 84: 78-87.

Shevde, N.K., Bendixen, A.C., Dienger, K.M., and Pike, J.W. (2000). Estrogens suppress RANK ligand-induced osteoclast differentiation via a stromal cell independent mechanism involving c-Jun repression. Proc. Natl. Acad. Sci. 97(14): 7829-7834.

Surh, Y.J., Chun, K.S., Cha, H.H., Han, S.S., Keum, Y.S., Park, K.K., and Lee, 
S.S. (2001). Molecular mechanisms underlying chemopreventive activities of anti-inflammatory phytochemicals: down-regulation of COX-2 and iNOS through suppression of NF-KB activation. Mutat. Res. 480: 243-268.

Takatsuna, H., Asagiri, M., Kubota, T., Oka, K., Osada, T., Sugiyama, C., and Umezawa, K. (2005). Inhibition of RANKL-Induced Osteoclastogenesis by (-)-DHMEQ, a Novel NF-KB Inhibitor, Through Downregulation of NFATc1. J. Bone Miner. Res. 20(4): 653-662.

Takayanagi, H., Kim, S., Koga, T., Nishina, H., Isshiki, M., Yoshida, H., and Wagner, E.F. (2002). Induction and activation of the transcription factor NFATc1 (NFAT2) integrate RANKL signaling in terminal differentiation of osteoclasts. Dev. Cell 3(6): 889-901.

Teitelbaum, S.L. (2000). Bone resorption by osteoclasts. Science 289(5484):
1504-1508.

Tracey, D., Klareskog, L., Sasso, E.H., Salfeld, J.G., and Tak, P.P. (2008). Tumor necrosis factor antagonist mechanisms of action: a comprehensive review. Pharmacol. Ther. 117(2): 244-279.

Xu, C., Yang, C., Yin, Y., Liu, J., and Mine, Y. (2012). Phosphopeptides (PVHs) from hen egg yolk phosvitin exert anti-inflammatory activity via modulation of cytokine expression. J. Funct. Foods 4(4): 718-726.

Young, D., Nau, F., Pasco, M., and Mine, Y. (2011). Identification of hen egg yolk-derived phosvitin phosphopeptides and their effects on gene expression profiling against oxidative stress-induced Caco-2 cells. J. Agric. Food Chem. 59(17): 9207-9218.

Zhang, W., and Liu, H.T. (2002). MAPK signal pathways in the regulation of cell proliferation in mammalian cells. Cell Res. 12(1): 9. 